\title{
Laparoscopic Diaphragmatic Plication in Patients with Unilateral Diaphragm Paralysis with Thoracoscopic Verification
}

\author{
Willem N. Welvaart ${ }^{1,2}$, Kieng H. Ong ${ }^{1}$, Marinus A. Paul ${ }^{2}$, Anton Vonk-Noordegraaf ${ }^{2}$, \\ Coen A. C. Ottenheijm ${ }^{2}$ \\ ${ }^{1}$ Department of Surgery, Rivierenland Hospital, Tiel, The Netherlands; ${ }^{2}$ Department of Surgery, Pulmonology and Physiology, VU \\ Medical Center, Amsterdam, The Netherlands. \\ Email: w.welvaart@zrt.nl
}

Received July 25 ${ }^{\text {th }}$, 2012; revised August 30 ${ }^{\text {th }}$, 2012; accepted September $10^{\text {th }}$, 2012

\begin{abstract}
Diaphragm eventration or paralysis causes elevation of the ipsilateral diaphragm. Some patients have symptoms. The most common symptom is dyspnea. In symptomatic patients with unilateral diaphragm paralysis and dyspnea disproportionate to the degree of physical activity, diaphragm plication is the treatment of choice to relieve dyspnea. We report on our experience with laparoscopic plication of a paralysed hemidiaphragm. The laparoscopic approach is an attractive surgical alternative for the treatment of phrenic nerve palsy. This technique combines the advantage of an excellent field of vision during surgery with a fast postoperative recovery and early discharge from hospital. In accordance to recent literature, we have successfully applied the laparoscopic approach to treat our patients who suffered from a unilateral paralysis of the diaphragm. Preoperative symptoms of exertional dyspnea and orthopnea were significantly reduced.
\end{abstract}

Keywords: Diaphragm Paralysis; Dyspnea; Diaphragm Plication; Laparoscopy

\section{Introduction}

An elevated diaphragm due to paralysis is an uncommon condition. It may be an incidental finding in asymptomatic patients, but sometimes it is the cause of orthopnea and dyspnea on excertion. In the latter patients the clinical condition can be improved by plication, limiting the paradoxal movement on respiration. For the aetiology, a distinction is made between eventration and acquired paralysis, but symptoms and treatment for both conditions are similar

A variety of open and minimally invasive diaphragm plication techniques have been described, but all are hampered by posthoracotomy pain or limited vision.

A recent patient, who became symptomatic after a phrenic nerve palsy following surgery for a cervical spinal stenosis, made us change our approach. This 67-yearold male was found to have a hepatodiaphragmatic interposition of the large intestine, i.e. Chilaiditi's sign, as well (Figure 1). Because of this interposition, we chose a laparoscopic approach, to minimise the risk of intestine perforation or compression. After repositioning of the colon the diaphragm was plicated. Visibility was excellent and the patient experienced minimal discomfort.

\section{Technique}

Our patient was intubated with a double lumen tube. His position was supine, with the surgeon positioned between the legs. The first trocar was placed subumbilically. After CO2 insufflation of the abdomen two other trocars were placed, one in the midline between xyphoid and umbilicus, the other in the upper ipsilateral quadrant of the abdomen.

We deliberately perforated the dome of the diaphragm, using a diathermic hook, to create an ipsilateral pneumothorax. This causes the diaphragm to fall down. By

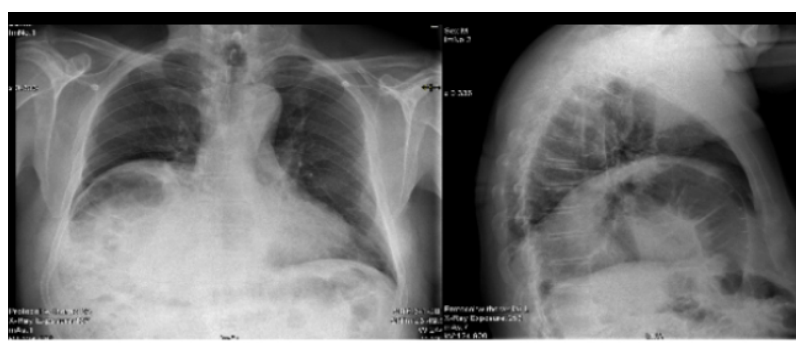

Figure 1. Preoperative chest radiographs showed an elevated position of the right hemi-diaphragm with also Chilaiditi's Sign. 
opening the diaphragm, the $\mathrm{CO}_{2}$ inflated abdominal cavity is partly desufflated in the thoracic cavity and a steady-state of intra-abdominal pressure and intrathoracic pressure is obtained. Because of this steady-state situation, the diaphragm can be brought down without any resistance. Before opening the diaphragm, ipsilateral lung collaps is obtained by using double lumen tube intubation. Through the deliberately opened diaphragm, good visibility is obtained for inspection with the abdominal camera of the thoracic cavity. If any adhesions are expected, care must be taken by opening of the diaphragm. In case of adhesions, a VATS-procedure provides an appropriate alternative for adhesiolysis before performing the plication technique.

A percutaneously inserted retention stitch was used to pull down the diaphragm even further (Figure 2). The percutaneous retention stitch is passed through one of the trocards, and after good bite of the diaphragm again through the trocard out of the abdominal cavity. After placing a sufficient retention stitch, the trocard is removed and placed in its original place with the stitch beside it. With this technique there is no gas leak to be expected. An alternative method is placing the retention stitch through the abdominal wall, which also minimized the risk of a gas leak.

Diaphragmatic plications were easily created due to the tension free hemidiaphragm (Figure 1), using running nonabsorbable sutures. Simple plication of the diaphragm involves mobilizing the muscle in a radial direction while creating pleats in the anteromedial and posteromedial direction using full-thickness horizontal mattress sutures. The created small diaphragm perforation was closed by incorporating it in one of the plications.

Following plication, the retention stitch was cut and as a result of the insufflated abdominal cavity we could evaluate the effective result of plication during surgery

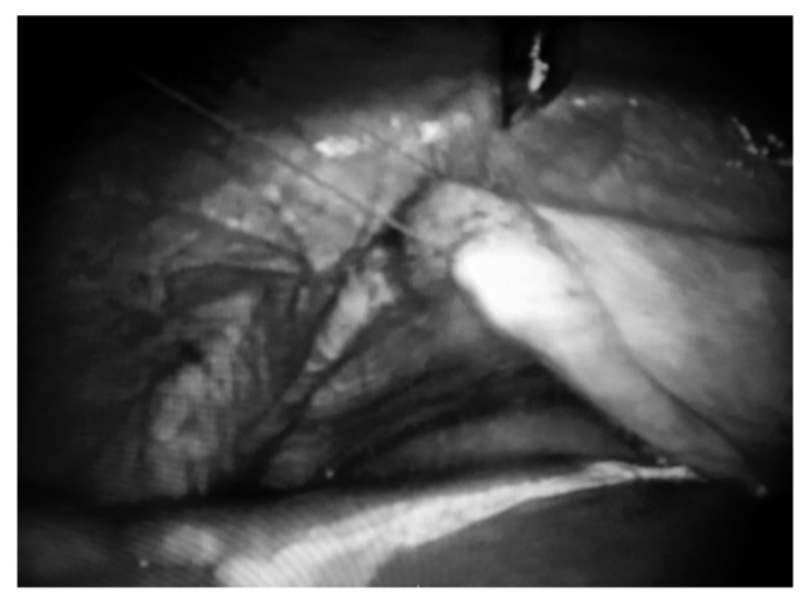

Figure 2. After perforation the dome of the diaphragm, diaphragmatic plications were easily created due to the tension free hemidiaphragm.
(Figure 3). Subsequently we performed a thoracoscopy to evaluate the plicated hemidiaphragm from the upper site and to exclude any pulmonary injury (Figure 4). This thoracoscopy port is then used to insert a chest tube. Removal of this chest tube was within the 24 hours.

Following this first successful treatment, the laparoscopic approach has become our standard procedure for treatment of patients with hemidiaphragm paralysis (Figure 5).

So far seven patients have been treated (Tables $\mathbf{1}$ and 2). All were discharged within three postoperative days with minimal pain. In all the preoperative symptoms of exertional dyspnea and orthopnea were significantly reduced.

\section{Discussion}

The first plication was performed in 1923 by Morrison [1], although this treatment was already suggested seven years earlier [2]. Since then, a variety of open and minimally invasive diaphragm plication techniques have been described to reduce symptomatic dysfunctional diaphragm

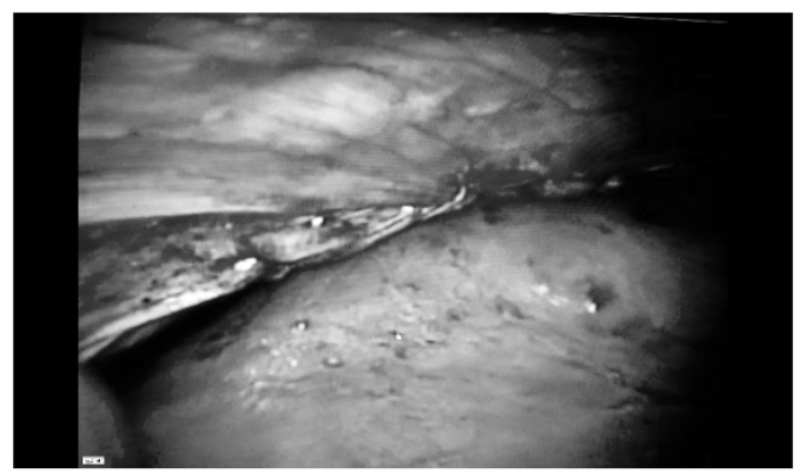

Figure 3. After plication of the diaphragm, a potential interposition of the large intestine was prevented.



Figure 4. After plication of the diaphragm we used video assisted thoracoscopic surgery to evaluate the plicated hemidiaphragm. 
Table 1. Patients characteristics.

\begin{tabular}{|c|c|c|c|c|c|c|c|}
\hline Sex & pt & Age & Side & BMI & $\begin{array}{c}\text { Duration } \\
\text { of paralysis }\end{array}$ & Aetiology & Comorbidity \\
\hline $\mathrm{M}$ & $\# 1$ & 67 & Right & 29.4 & 19 years & Cervicalspine surgery '91 & Chilaiditi Syndrome, TIA, CVA, appendectomy, umbilical hernia \\
\hline $\mathrm{F}$ & $\# 2$ & 79 & Left & 25.3 & Unknown & Unknown & Hypertension, multi-nodulargoiter \\
\hline M & \#3 & 66 & Right & 24.5 & Unknown & Unknown & $\begin{array}{l}\text { Lumbar disc herniation, trauma of the right shoulder, cervical } \\
\text { spine stenosis }\end{array}$ \\
\hline $\mathrm{F}$ & $\# 4$ & 82 & Right & 30.0 & Unknown & Unknown & NissenFundoplication, left sided nervus laryngeus recurrens \\
\hline $\mathrm{F}$ & $\# 5$ & 52 & Right & 23.9 & 1 year & $\begin{array}{l}\text { Vascular intervention in } \\
\text { the neck }\end{array}$ & $\begin{array}{l}\text { Pulmonary hpertension, right sided arteriovenous malformation } \\
\text { in the neck and thoracic wall }\end{array}$ \\
\hline M & $\# 6$ & 64 & Right & 32.0 & Unknown & Unknown & Cervical spine stenosis, COPD \\
\hline $\mathrm{F}$ & \#7 & 78 & Right & 24.8 & 33 years & $\begin{array}{c}\text { Pulmonary } \\
\text { sequestrectomy }\end{array}$ & $\begin{array}{l}\text { Right sided pulmonary sequestration operation, cercical spine } \\
\text { stenosis, obesitas }\end{array}$ \\
\hline
\end{tabular}

Abbreviations: $\mathrm{M}=$ male; $\mathrm{F}$ = female; $\mathrm{BMI}=$ body mass index.

Table 2. Spirometry, pre- and post-operative changes.

\begin{tabular}{cccccc}
\hline \multirow{2}{*}{ Sex } & \multirow{2}{*}{$\mathbf{p t}$} & \multicolumn{2}{c}{ Pre-operative } & \multicolumn{2}{c}{ Post-operative } \\
\cline { 3 - 6 } & & FEV1 (\% predicted) & FEV1/VC (\%) & FEV1 (\% predicted) & FEV1/VC (\%) \\
\hline M & $\# 1$ & $1.98(68 \%)$ & $69 \%$ & $2.15(75 \%)$ & $68 \%$ \\
F & $\# 2$ & $1.88(98 \%)$ & $66 \%$ & $1.89(99 \%)$ & $71 \%$ \\
M & $\# 3$ & $1.79(57 \%)$ & $78 \%$ & $1.94(62 \%)$ & $87 \%$ \\
F & $\# 4$ & $1.41(93 \%)$ & $89 \%$ & $1.28(84 \%)$ & $76 \%$ \\
F & $\# 5$ & $0.59(19 \%)$ & $74 \%$ & $1.69(56 \%)$ & $81 \%$ \\
M & $\# 6$ & $1.87(53 \%)$ & $56 \%$ & $2.82(82 \%)$ & $68 \%$ \\
F & $\# 7$ & $0.95(49 \%)$ & $64 \%$ & $1.26(64 \%)$ & $78 \%$ \\
\hline
\end{tabular}

Abbreviations: FEV1 = forced expiratory volume in 1 second; $\mathrm{VC}=$ vital capacity.

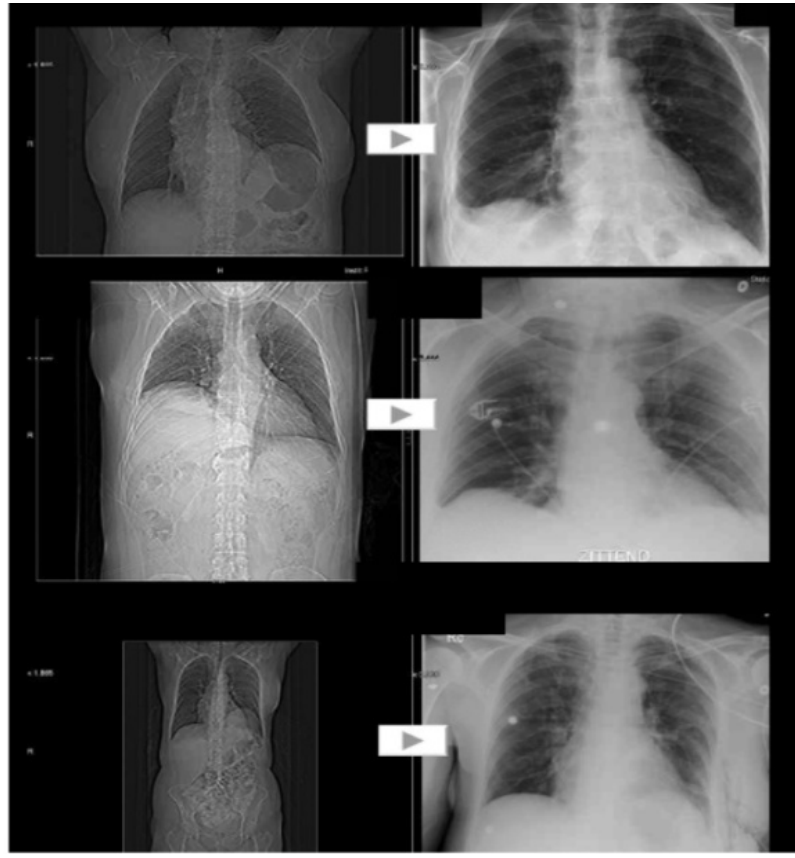

Figure 5. Examples of preoperative and postoperative chest radiographs of some patients with hemidiaphragm paralysis. excursion during respiration [3].

The aim of the operation is to relieve dyspnea, therefore it is indicated in symptomatic patients only. A waiting time is often advised in phrenic nerve injury after cardiac surgery, because nerve function may improve over time. However, many patients have already longstanding and disabling symptoms and spontaneous recovery in this group is not to be expected. Therefore, in symptomatic patients with unilateral diaphragm paralysis we proceed to surgical plication. The plication immobilizes the diaphragm in a lower position and reduces the paradoxical excursions, making it easier for the accessory respiratory muscles to produce a negative inspiratory pressure.

In literature relative contra-indications to diaphragm plication are morbid obesity and certain neuromuscular disorders [3]. In our experiences, in morbid obesity the laparoscopic technique may be the approach of choice.

It is known that postoperative pain after thoracotomy or thoracoscopy can be significant with the possibility of evolvement to chronic neuralgia. The laparoscopic approach avoids this complication. Evaluation of laparoscopic diaphragmatic plication for hemidiaphragmatic 
paralysis or eventration demonstrated significant shortterm and midterm improvements in respiratory quality of life and pulmonary function test results. This approach represents a potential paradigm shift in the surgical management of hemidiaphragmatic paralysis or eventration [4]. Also in our experience the laparoscopic approach in plication of the diaphragm causes minimal pain. In our opinion this laparoscopic approach should be the first choice. We do realise that due to the rarity of the disease, there is only a limited number of patients that will benefit from this approach. However, these first results are that promising that we think our technique provides an interesting alternative for the conventional treatment of this group of patients.

\section{Conclusions}

Laparoscopic diaphragmatic plication combines excellent relief of symptoms with minimal morbidity.

In accordance to recent literature, we have success- fully applied the laparoscopic approach to treat our patients who suffered from a unilateral paralysis of the diaphragm.

\section{REFERENCES}

[1] J. M. Morrison, "Eventration of Diaphragm Due to Unilateral Phrenic Nerve Paralysis,” Archives of Radiology and Electrotherapy, Vol. 28, 1923, pp. 72-75.

[2] H. G. Wood, "Eventration of the Diaphragm," Surgery, Gynecology \& Obstetrics, Vol. 23, 1916, pp. 244-248.

[3] S. S. Groth and R. S. Andrade, "Diaphragmatic Eventration,” Thoracic Surgery Clinics, Vol. 19, No. 4, 2009, pp. 511-519.

[4] S. S. Groth, N. M. Rueth, T. Kast, J. D’Cunha, R. F. Kelly, M. A. Maddaus and R. S. Andrade, "Laparoscopic Diaphragmatic Plication for Diaphragmatic Paralysis and Eventration: An Objective Evaluation of Short-Term and Midterm Results," Journal of Thoracic and Cardiovascular Surgery, Vol. 139, No. 6, 2010, pp. 1452-1456. 